\title{
Domain Structure of Bulk and Thin-Film Ferroelectrics By Transmission Kikuchi Diffraction
}

\author{
Matthew J. Burch ${ }^{1}$, David T. Harris ${ }^{1}$, Chris M. Fancher ${ }^{1}$, Jon-Paul Maria ${ }^{1}$, and Elizabeth C. Dickey ${ }^{1}$. \\ ${ }^{1}$ North Carolina State University, Materials Science and Engineering, Raleigh, NC USA.
}

Non-linear dielectrics are of great interest due to their integration into modern electronics, including tunable and memory devices [1]. Recently, much work has been invested into obtaining bulk-like properties of dielectric thin films at lower processing temperatures than are generally required for maximum performance. In particular, Harris et. al. has shown impressive dielectric properties of thin films at processing temperatures as low as $900^{\circ} \mathrm{C}$ for barium titanate $\left(\mathrm{BaTiO}_{3}\right)$, contrasted with bulk, where similar properties would need $1250^{\circ} \mathrm{C}$ or higher processing temperatures [2].

While dielectric properties (permittivity, tunability, etc.) of ferroelectric oxides are generally considered to be the result of the extrinsic effect of domain wall motion, ferroelectric domains are rarely observed in small grained $(<0.4 \mu \mathrm{m})$ samples [3]. Thus, if these domains are present, their formation and structure is not well defined or understood. This study utilizes modern day diffraction and imaging techniques to understand nano-domains and their impact on the dielectric properties of small-grained bulk and thin film dielectrics.

Transmission Kikuchi diffraction (TKD) is a relatively new technique utilizing an electron backscatter diffraction (EBSD) detector in a scanning electron microscope (SEM), where electrons are transmitted through a thin sample and captured similarly to traditional EBSD, as shown in figure 1. This geometry allows for significantly enhanced resolution $(<10 \mathrm{~nm})$ compared to traditional EBSD $(\sim 80 \mathrm{~nm})$ and allows for examination of domain structure in samples with grain sizes too small for traditional EBSD. In this study, TKD was used to identify and characterize the domain structure of small-grained samples composed of thin-film $\mathrm{BaTiO}_{3}$ or lead zirconate titanate $\left(\mathrm{Pb}(\mathrm{Zr}, \mathrm{Ti}) \mathrm{O}_{3}\right)$. Furthermore, we wish to use these studies to find the fundamental limit to the spatial and strain resolution of TKD.

Figure 2 shows an example TKD band-contrast image and inverse pole figure (IPF) maps of bulk $\mathrm{Pb}(\mathrm{Zr}, \mathrm{Ti}) \mathrm{O}_{3}$, with the domain structure clearly present in both images. A band contrast image is generated when the intensity of the Kikuchi bands is compared to the overall strength of the TKD pattern. Areas where the Kikuchi bands are less intense, such as grain boundaries, defective areas, or domain boundaries, will appear darker than areas of strongly diffracting Kikuchi bands, such as in the middle of a grain or domain. The striped domains shown in figure 2 correspond to $90^{\circ}$ domains, as observed by both the IPF Z (grain orientations relative to out-of-plane direction) and IPF Y (grain orientation relative to the vertical in-plane direction) images. This investigation shows these striped domains are on the order of $60-80 \mathrm{~nm}$ in width.

Figure 3 shows the TKD patterns within a single grain of a large grained $\mathrm{BaTiO}_{3}$ thin film sample. The orientation change from the first and second TKD patterns is consistent with a $90^{\circ}$ domain. These TKD patterns demonstrate the extreme sensitivity of TKD as a characterization technique for domain structures in ferroelectrics as the c/a ratio of $\mathrm{BaTiO}_{3}$ is only $\sim 1.005$ [4]. 


\section{References:}

[1] K. Tagantsev, et. al., Journal of Electroceramics 11 (2003), p. 5

[2] D. T. Harris, et. al., Applied Physics Letters 103 (2013), p. 012904

[3] G. Arlt and D. Hennings, Journal of Applied Physics 58 (1985), p. 1619

[4] The authors acknowledge funding from the Center for Dielectrics and Piezoelectrics at the North Carolina State University. This material is based upon work supported by the National Science Foundation Graduate Research Fellowship for M. J. Burch under Grant No. DGE-0946818. The authors acknowledge the use of the Analytical Instrumentation Facility (AIF) at North Carolina State University, which is supported by the State of North Carolina and the National Science Foundation.

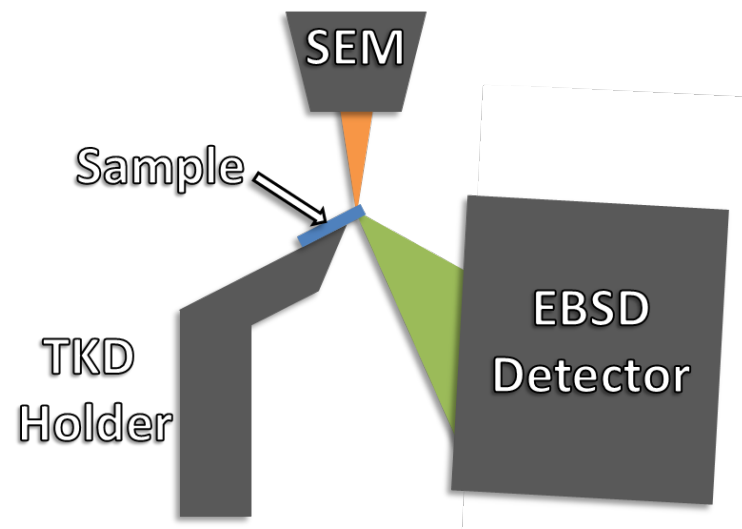

Figure 1: Schematic diagram of transmission Kikuchi diffraction (TKD). The thin $(<150 \mathrm{~nm})$ sample is oriented so the incoming electron beam is transmitted through the thin sample, which is then collected by the EBSD detector.
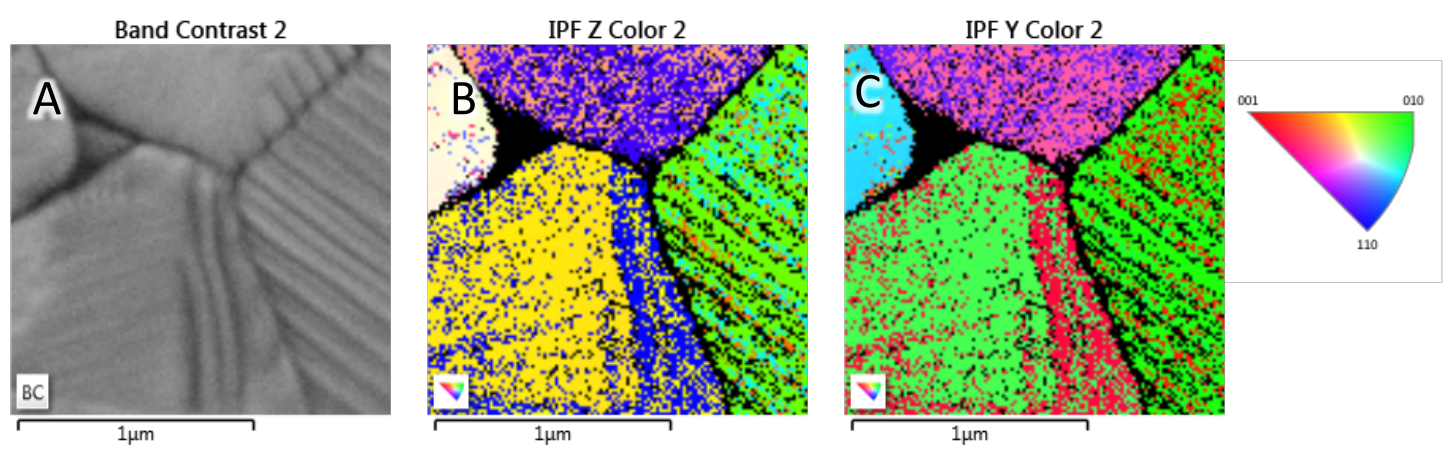

Figure 2: (A) Band contrast image of bulk PZT samples with the domains clearly evident. The following images are IPF images, with the $90^{\circ}$ domains clearly evident for both the Z-(B) and Y-(C) relative orientation IPF images.
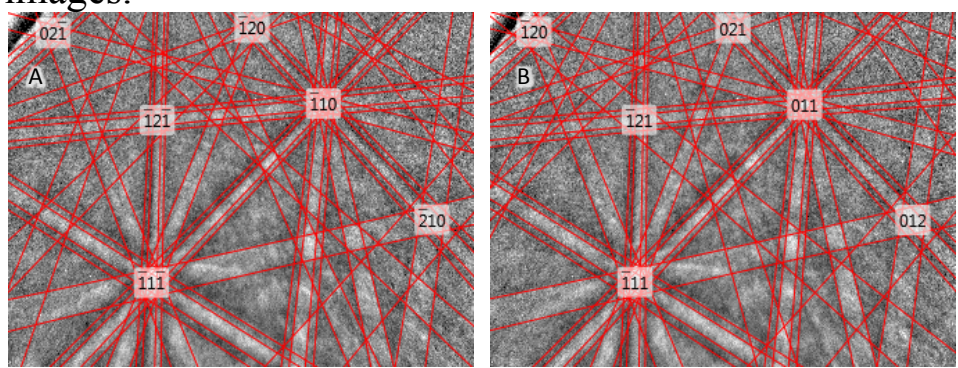

Figure 3: EBSD patterns of a within a single grain of a barium titanate thin film, showing a change in orientation that is consistent with a $90^{\circ}$ domain. 